\title{
Nonlinear Qubit Transformations
}

\author{
Lucien Hardy and David D. Song \\ Centre for Quantum Computation \\ Clarendon Laboratory, Department of Physics \\ University of Oxford, Parks Road, Oxford OX1 3PU, U.K.
}

\begin{abstract}
We generalise our previous results of universal linear manipulations [Phys. Rev. A 63, $032304(2001)$ ] to investigate three types of nonlinear qubit transformations using measurement and quantum based schemes. Firstly, nonlinear rotations are studied. We rotate different parts of a Bloch sphere in opposite directions about the $z$-axis. The second transformation is a map which sends a qubit to its orthogonal state (which we define as ORTHOG). We consider the case when the ORTHOG is applied to only a partial area of a Bloch sphere. We also study nonlinear general transformation, i.e. $(\vartheta, \varphi) \rightarrow(\vartheta-\alpha, \varphi)$, again, applied only to part of the Bloch sphere. In order to achieve these three operations, we consider different measurement preparations and derive the optimal average (instead of universal) quantum unitary transformations. We also introduce a simple method for a qubit measurement and its application to other cases.
\end{abstract}

\section{Introduction}

In recent years interests in the field of quantum computation has grown rapidly (see [1, 2, 3] for reviews). For a deeper understanding of the fundamental principles and limitations of quantum computing, it is important to investigate optimal operations for unknown states. In a Bloch sphere notation, an unknown qubit has the following form,

$$
|\psi(\vartheta, \varphi)\rangle=\cos \frac{\vartheta}{2}|0\rangle+e^{i \varphi} \sin \frac{\vartheta}{2}|1\rangle
$$


There have been a number of studies to find optimal manipulations of quantum states. Bužek and Hillery introduced [4, 5] a universal quantum cloner such that, given a single unknown qubit, it produces two approximate copies of the input qubit. Subsequently, Gisin and Massar generalised it [6] to a $N$ to $M$ cloner. These cloners have been proven to be optimal by various researchers [7, 8, 9, 6]. A universal quantum entangler has also been introduced in [10]. It entangles two qubits where one is unknown while the other is a known reference state. Moreover, Mor and Terno introduced a universal quantum disentangler [11, 12, 13] (also see [14]). They considered a bipartite state and its transformation into a product state of the reduced density matrices of the two subsystems. Bužek and Hillery considered [15] a slight different disentangler which extracts an unknown qubit entangled with a reference state. Recently, universal-NOT (U-NOT) transformation has been studied in [16, 17, 18]. The perfect operation of $|\psi\rangle \rightarrow\left|\psi^{\perp}\right\rangle$ is not allowed due to its anti-unitarity. In a single qubit case, the fidelity of imitating such operation was shown to be $2 / 3$. This is same as the measurement fidelity which is the optimal efficiency of measuring an unknown qubit. Universality of these transformations imply that the operations yield the same fidelity regardless of the input states. In [19], more general universal manipulations of a qubit were derived by the present authors. We considered a linear transformation,

$$
|\psi(\vartheta, \varphi)\rangle \rightarrow|\psi(\vartheta-\alpha, \varphi-\beta)\rangle
$$

where the transformation of $\varphi$ by $\beta$ can always be done by unitary rotation about the $z$-axis. In performing the transformation of $\vartheta$, there are two types of approaches we could take. One, which we call the measurement scheme, is to measure the qubit then prepare another qubit according to the transformation in (2). The other approach, which we call the quantum scheme, is by a quantum unitary transformation on the system plus an ancilla. In case of the quantum approach, we found the procedures of unitary transformation that optimise fidelity fall into two classes depending on the phase angle change $\alpha$. For $0 \leq \alpha \leq \pi / 2$ the best way is simply an identity map while for $\pi / 2 \leq \alpha \leq \pi$, Bužek et al.'s U-NOT gate is the optimal transformation.

The transformations we will consider in this paper do not respect the symmetry of the Bloch sphere and therefore it does not make sense to impose universality. Hence, we will consider optimisation of the average fidelity without imposing that the fidelity be the same for each input state.

We also want to define an operation which sends a qubit to its orthogonal state,

$$
\text { ORTHOG : }|\psi\rangle \rightarrow\left|\psi^{\perp}\right\rangle
$$

When we impose universality on the operation ORTHOG, it would correspond to the Bužek et al.'s U-NOT gate.

In this paper, we attempt to generalise the linear transformation in (2) to various nonlinear cases. Gisin has proposed [20] a rather peculiar rotation where upper and lower 
hemispheres of a Bloch sphere are rotated in opposite directions about the $z$-axis. We study both measurement and quantum approaches of this type of nonlinear map and also consider other types of nonlinear operations. Our results are summarised as follows;

- We study a nonlinear transformation as shown in figure 2, which rotates different parts of a Bloch sphere in opposite directions about the $z$-axis. When the two parts are upper and lower hemispheres, the optimal map is either identity or the unitary rotation by $\pi$. We find the average optimal transformations for general nonlinear rotations.

- Nonlinear ORTHOG gates are studied. We investigate averaged (instead of universal) ORTHOG transformations that are applied only to a partial area of a Bloch sphere as shown in figure 6. Bužek et al.'s U-NOT gate appears as a one particular case when the transformation is applied to the whole Bloch sphere and universality is imposed. In general, we show the optimal map is either identity or the unitary operation $\sigma_{x}=\left(\begin{array}{ll}0 & 1 \\ 1 & 0\end{array}\right)$, a Pauli matrix.

- We consider nonlinear general transformations (i.e. $(\vartheta, \varphi) \rightarrow(\vartheta-\alpha, \varphi))$ as shown in figure 10. Unlike the ORTHOG-gate, the averaged general transformation has higher fidelity than the universal manipulation of a qubit in general. We show the optimal operations for different values of $\alpha$.

- We also show a simple way of measuring an unknown qubit, measurement-based U-NOT and general linear transformation while obtaining the same fidelities as in the case of a conventional method.

In sect. 2, we review a traditional method of qubit measurement and show a simpler way to achieve the same fidelity. This particular way also works equally well for measurement based U-NOT and the general linear transformation in (2). In sect. 3, we study Gisin's proposed nonlinear rotations. In sect. 4 and 5 , we study nonlinear transformation of ORTHOG and the general transformation in (2) where the operations are applied only to chosen areas of a Bloch sphere.

\section{Measuring an Unknown Qubit}

In order to get maximum information about a single unknown qubit, we measure the qubit along any chosen basis $\left\{|\phi\rangle,\left|\phi^{\perp}\right\rangle\right\}[21$, 22]. If the result is $|\phi\rangle$, then we guess the qubit to be $|\phi\rangle$ and if the result is $\left|\phi^{\perp}\right\rangle$, then we guess $\left|\phi^{\perp}\right\rangle$. In density matrix terms, the state prepared is written as follows,

$$
\rho_{1}=|\langle\psi \mid \phi\rangle|^{2}|\phi\rangle\left\langle\left.\phi|+|\left\langle\psi \mid \phi^{\perp}\right\rangle\right|^{2} \mid \phi^{\perp}\right\rangle\left\langle\phi^{\perp}\right|
$$




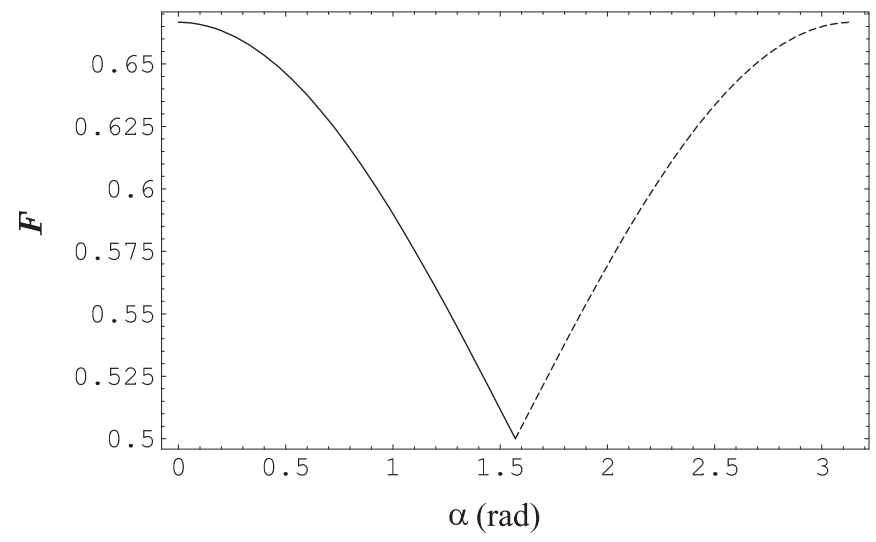

Figure 1: This graph shows the fidelity of measurement based transformation and the phase angle $\alpha$. The full line represents the preparation $\sigma_{1}$ and the dotted line is for $\sigma_{2}$. This result is identical to the usual measurement based schemes considered in [19.

Averaging over all possible $|\phi\rangle$ 's (assuming a uniform distribution over the Bloch sphere), we obtain $\overline{\rho_{1}}$. In order to obtain the fidelity, we take another integral of $\left\langle\psi\left|\overline{\rho_{1}}\right| \psi\right\rangle$ over possible inputs of $|\psi\rangle$. After the integration, the fidelity turns out to be $2 / 3$.

A simpler way of achieving the same result is as follows. Instead of (4), we measure onto the $\{|0\rangle,|1\rangle\}$ basis. As in the case of (击, if we obtain $|0\rangle$ then we guess the unknown state to be $|0\rangle$ and when $|1\rangle$ is obtained we guess $|1\rangle$. In a density matrix form, it is

$$
\sigma_{1}=|\langle\psi \mid 0\rangle|^{2}|0\rangle\left\langle\left. 0|+|\langle\psi \mid 1\rangle\right|^{2} \mid 1\right\rangle\langle 1|
$$

We don't need to take the average of $\sigma_{1}$ since we chose one particular basis $\{|0\rangle,|1\rangle\}$ instead of arbitrary basis. We then take the integral of $\left\langle\psi\left|\sigma_{1}\right| \psi\right\rangle$ over all possible input states $|\psi\rangle$ and we get the fidelity of $2 / 3$.

In [16, 17, 18, the U-NOT transformation has been discussed. A measurement-based U-NOT operation has been discussed in [17] which can be achieved as follows: If we get positive with a guessed state, we prepare the orthogonal state to the guessed state and when the result is negative we prepare the guessed state, i.e.

$$
\rho_{2}=|\langle\psi \mid \phi\rangle|^{2}\left|\phi^{\perp}\right\rangle\left\langle\left.\phi^{\perp}|+|\left\langle\psi \mid \phi^{\perp}\right\rangle\right|^{2} \mid \phi\right\rangle\langle\phi|
$$

The result obtained was same as the measurement fidelity of $2 / 3$. We can also use a similar method to that in (5). We measure onto the $\{|0\rangle,|1\rangle\}$ basis and when we get $|0\rangle$ we prepare $|1\rangle$ and for $|1\rangle,|0\rangle$ is prepared. This gives

$$
\sigma_{2}=|\langle\psi \mid 0\rangle|^{2}|1\rangle\left\langle\left. 1|+|\langle\psi \mid 1\rangle\right|^{2} \mid 0\right\rangle\langle 0|
$$

After taking an integral of $\left\langle\psi\left|\sigma_{2}\right| \psi\right\rangle$ over all $|\psi\rangle$, we again obtain the same fidelity $2 / 3$ as in the case of $\rho_{2}$. In [19], we considered a general linear transformation

$$
(\vartheta, \varphi) \rightarrow(\vartheta-\alpha, \varphi)
$$


Even in the case of transformation (8), two density matrices $\sigma_{1}$ and $\sigma_{2}$ yield the same results as obtained in [19]. For $0 \leq \alpha \leq \pi / 2, \sigma_{1}$ in (5) and for $\pi / 2 \leq \alpha \leq \pi, \sigma_{2}$ give the fidelity as shown in figure 1. This fidelity is same as the ones obtained by $\rho_{1}$ in (里) and $\rho_{2}$ in $(6)$.

We have shown that even with a specific basis $\{|0\rangle,|1\rangle\}$, we obtain the same results for measurement of arbitrary states, the U-NOT and the general linear transformation cases. However we will show in the following sections, this particular choice of basis does not give the same results for other measurement based nonlinear transformations.

\section{Nonlinear Rotations}

It is well known that rotations of a qubit about the $z$-axis by the angle $\beta$ can be achieved unitarily with the following operator,

$$
R_{z}(\beta)=\left(\begin{array}{cc}
e^{-i \beta / 2} & 0 \\
0 & e^{i \beta / 2}
\end{array}\right)
$$

Gisin has proposed [20] a rather different rotation of a qubit. For some inputs $|\psi(\vartheta, \varphi)\rangle$ we rotate by the angle $\beta$ about the $z$-axis and for different $(\vartheta, \varphi)$ we rotate by $-\beta$. Suppose given an unknown state, we want to transform it as follows

$$
\left\{\begin{array}{l}
0 \leq \vartheta<\delta, \quad|\psi(\vartheta, \varphi)\rangle \rightarrow|\psi(\vartheta, \varphi+\beta)\rangle \\
\delta \leq \vartheta \leq \pi, \quad|\psi(\vartheta, \varphi)\rangle \rightarrow|\psi(\vartheta, \varphi-\beta)\rangle
\end{array}\right.
$$

In a Bloch sphere, this can be drawn as shown in figure 2. We consider two different approaches in achieving the transformations in (10), measurement and quantum based schemes. We first consider a measurement scheme.

Measurement Scheme Let us consider the case when $\delta=\pi / 2$. The first thing we could try is to use a similar method to that in (田). That is, we measure onto the guessed basis $\left\{|\phi\rangle,\left|\phi^{\perp}\right\rangle\right\}$ (where $|\phi\rangle$ is chosen to be in the upper hemisphere) and rotate by $+\beta$ if the result is positive and when $\left|\phi^{\perp}\right\rangle$ is obtained we rotate by $-\beta$. In this case, we have the density matrix,

$$
\rho_{3}=|\langle\psi \mid \phi\rangle|^{2}|\phi(\mu, \nu+\beta)\rangle\left\langle\left.\phi(\mu, \nu+\beta)|+|\left\langle\psi \mid \phi^{\perp}\right\rangle\right|^{2} \mid \phi^{\perp}(\mu, \nu-\beta)\right\rangle\left\langle\phi^{\perp}(\mu, \nu-\beta)\right|
$$

Then we need to take the average of $\rho_{3}$ for all possible $|\phi(\mu, \nu)\rangle$ 's where $0 \leq \mu \leq \pi / 2$ and $0 \leq \nu \leq 2 \pi$. If we take an example where $\beta=\pi / 3$, then the fidelity is obtained as

$$
\begin{aligned}
F & =\frac{1}{4 \pi} \int_{0}^{2 \pi} \int_{0}^{\delta=\pi / 2}\left\langle\psi(\vartheta, \varphi+\pi / 3)\left|\overline{\rho_{3}}\right| \psi(\vartheta, \varphi+\pi / 3)\right\rangle \sin \vartheta d \vartheta d \varphi \\
& +\frac{1}{4 \pi} \int_{0}^{2 \pi} \int_{\delta=\pi / 2}^{\pi}\left\langle\psi(\vartheta, \varphi-\pi / 3)\left|\overline{\rho_{3}}\right| \psi(\vartheta, \varphi-\pi / 3)\right\rangle \sin \vartheta d \vartheta d \varphi=.58333 \ldots
\end{aligned}
$$



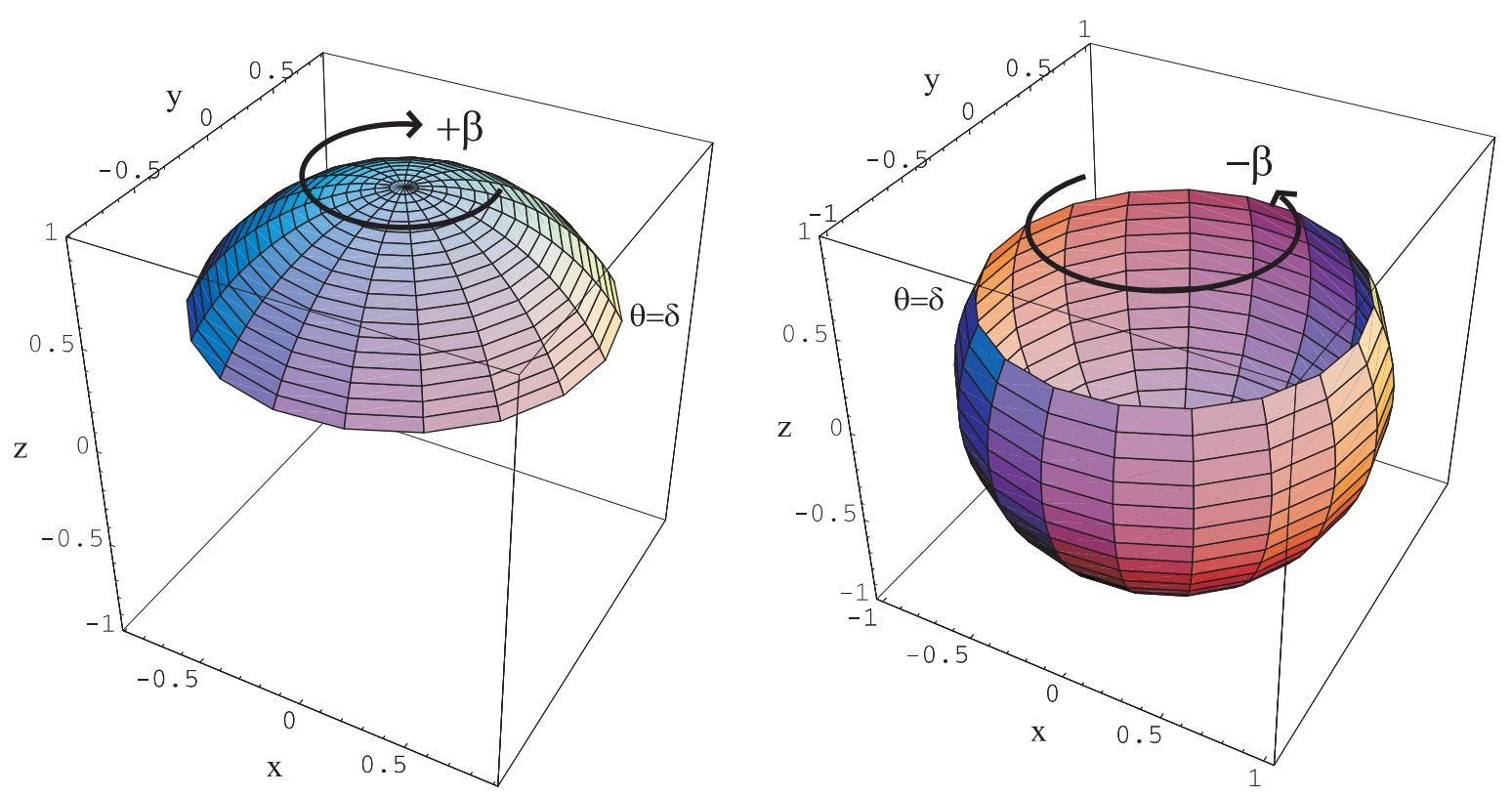

Figure 2: This figure shows nonlinear rotation of a Bloch sphere of an unknown qubit. For $\vartheta>\delta$, we rotate by $+\beta$ while for $\vartheta \leq \delta$ we rotate the qubit by $-\beta$ where $0 \leq \delta \leq \pi$.

where $\overline{\rho_{3}}$ is the averaged $\rho_{3}$. Can we do any better? Let us consider another preparation, $\rho_{1}$ in (田), i.e. we do not rotate by $+\beta$ or $-\beta$. Using the equation in (12), instead of $\overline{\rho_{3}}$ we put $\overline{\rho_{1}}$, then the fidelity is obtained as $.6111 \ldots$.

Let us consider another method that we considered in sect. 2, i.e. with $\sigma_{1}$ in (5). The fidelity is obtained as

$$
\begin{aligned}
F & =\frac{1}{4 \pi} \int_{0}^{2 \pi} \int_{0}^{\delta=\pi / 2}\left\langle\psi(\vartheta, \varphi+\beta)\left|\sigma_{1}\right| \psi(\vartheta, \varphi+\beta)\right\rangle \sin \vartheta d \vartheta d \varphi \\
& +\frac{1}{4 \pi} \int_{0}^{2 \pi} \int_{\delta=\pi / 2}^{\pi}\left\langle\psi(\vartheta, \varphi-\beta)\left|\sigma_{1}\right| \psi(\vartheta, \varphi-\beta)\right\rangle \sin \vartheta d \vartheta d \varphi=\frac{2}{3}
\end{aligned}
$$

This result is also independent of the values $\beta$ 's since the phase factor is cancelled in $\left\langle\psi(\vartheta, \varphi+\beta)\left|\sigma_{1}\right| \psi(\vartheta, \varphi+\beta)\right\rangle$ and $\left\langle\psi(\vartheta, \varphi-\beta)\left|\sigma_{1}\right| \psi(\vartheta, \varphi-\beta)\right\rangle$. Also note that $2 / 3$ can be obtained for any $\delta$ as well. Since the quantity we are integrating is independent of $\beta$, therefore $\left\langle\psi(\vartheta, \varphi+\beta)\left|\sigma_{1}\right| \psi(\vartheta, \varphi+\beta)\right\rangle=\left\langle\psi(\vartheta, \varphi-\beta)\left|\sigma_{1}\right| \psi(\vartheta, \varphi-\beta)\right\rangle$. Then we can put the two integrals in (13) together which would give the same result regardless of $\delta$ in a single integral. Therefore for any $\beta$ or $\delta$, we can obtain the fidelity of $2 / 3$ according to the transformation in (10) with the measurement preparation of $\sigma_{1}$ of (5).

Quantum Scheme We now discuss how we can achieve the transformation in (10) by unitary operations. One can see that when $\delta=0, \pi$ then it can be achieved perfectly by the unitary rotation about the $z$-axis. Let us consider the most general transformation 
on a single qubit. We consider a unitary evolution on the single qubit and some ancilla prepared in a known state $|Q\rangle$ (this is taken to be normalised). This then gives,

$$
\begin{aligned}
& |0\rangle|Q\rangle \rightarrow|1\rangle|A\rangle+|0\rangle|B\rangle \\
& |1\rangle|Q\rangle \rightarrow|0\rangle|\tilde{A}\rangle+|1\rangle|\tilde{B}\rangle
\end{aligned}
$$

where $|A\rangle,|\tilde{A}\rangle,|B\rangle,|\tilde{B}\rangle$ may not be normalised nor orthogonal to each other. From orthogonality and normalisation conditions of (14), we have

$$
\begin{gathered}
|A|^{2}+|B|^{2}=1 \\
|\tilde{A}|^{2}+|\tilde{B}|^{2}=1 \\
\langle B \mid \tilde{A}\rangle+\langle A \mid \tilde{B}\rangle=0
\end{gathered}
$$

We consider an unknown state in a Bloch sphere as in (1) to go through the transformation in (14) and tracing over the ancilla states yields the following density matrix,

$$
\begin{aligned}
\rho^{\text {out }} & =|0\rangle\langle 1|\left(\cos ^{2} \frac{\vartheta}{2}\langle A \mid B\rangle+\sin ^{2} \frac{\vartheta}{2}\langle\tilde{B} \mid \tilde{A}\rangle+\cos \frac{\vartheta}{2} \sin \frac{\vartheta}{2} e^{i \varphi}\langle A \mid \tilde{A}\rangle+e^{-i \varphi} \cos \frac{\vartheta}{2} \sin \frac{\vartheta}{2}\langle\tilde{B} \mid B\rangle\right) \\
& +|1\rangle\langle 0|\left(\cos ^{2} \frac{\vartheta}{2}\langle B \mid A\rangle+\sin ^{2} \frac{\vartheta}{2}\langle\tilde{A} \mid \tilde{B}\rangle+\cos \frac{\vartheta}{2} \sin \frac{\vartheta}{2} e^{i \varphi}\langle B \mid \tilde{B}\rangle+e^{-i \varphi} \cos \frac{\vartheta}{2} \sin \frac{\vartheta}{2}\langle\tilde{A} \mid A\rangle\right) \\
& +|0\rangle\langle 0|\left(\cos ^{2} \frac{\vartheta}{2}|B|^{2}+\sin ^{2} \frac{\vartheta}{2}|\tilde{A}|^{2}+\cos \frac{\vartheta}{2} \sin \frac{\vartheta}{2} e^{i \varphi}\langle B \mid \tilde{A}\rangle+e^{-i \varphi} \cos \frac{\vartheta}{2} \sin \frac{\vartheta}{2}\langle\tilde{A} \mid B\rangle\right) \\
& +|1\rangle\langle 1|\left(\cos ^{2} \frac{\vartheta}{2}|A|^{2}+\sin ^{2} \frac{\vartheta}{2}|\tilde{B}|^{2}+\cos \frac{\vartheta}{2} \sin \frac{\vartheta}{2} e^{i \varphi}\langle A \mid \tilde{B}\rangle+e^{-i \varphi} \cos \frac{\vartheta}{2} \sin \frac{\vartheta}{2}\langle\tilde{B} \mid A\rangle\right)(17)
\end{aligned}
$$

Since we are considering a transformation which varies depending on the value of the input, we need to get the average value. That is, the fidelity can be obtained by averaging over $\vartheta$ and $\varphi$ instead of imposing a constraint such that it is independent of $\vartheta$ and $\varphi$ as in the universal transformation case.

Let us first consider the case when $\delta=\pi / 2$. The average fidelity is then

$$
\begin{aligned}
F & =\frac{1}{4 \pi} \int_{0}^{2 \pi} \int_{0}^{\pi / 2}\left\langle\psi(\vartheta, \varphi+\beta)\left|\rho^{\text {out }}\right| \psi(\vartheta, \varphi+\beta)\right\rangle \sin \vartheta d \vartheta d \varphi \\
& +\frac{1}{4 \pi} \int_{0}^{2 \pi} \int_{\pi / 2}^{\pi}\left\langle\psi(\vartheta, \varphi-\beta)\left|\rho^{\text {out }}\right| \psi(\vartheta, \varphi-\beta)\right\rangle \sin \vartheta d \vartheta d \varphi
\end{aligned}
$$

which comes out as

$$
F=\frac{1}{3}+\frac{1}{6}\left(|B|^{2}+|\tilde{B}|^{2}\right)+\frac{\cos \beta}{6}(\langle B \mid \tilde{B}\rangle+\langle\tilde{B} \mid B\rangle)
$$

We now would like to find the values of $|B|,|\tilde{B}|,\langle B \mid \tilde{B}\rangle$ and $\langle\tilde{B} \mid B\rangle$ such that the fidelity $F$ in (19) is the highest. We know $\cos \beta$ is positive for $0<\beta<\pi / 2$ while it's negative for $\pi / 2<\beta<\pi$. Therefore when $0 \leq \beta \leq \pi / 2,|B|^{2}=1=|\tilde{B}|^{2}$ and $\langle B \mid \tilde{B}\rangle=1=\langle\tilde{B} \mid B\rangle$ yields the highest fidelity which can be satisfied with $|B\rangle=|0\rangle,|\tilde{B}\rangle=|0\rangle$. This implies 


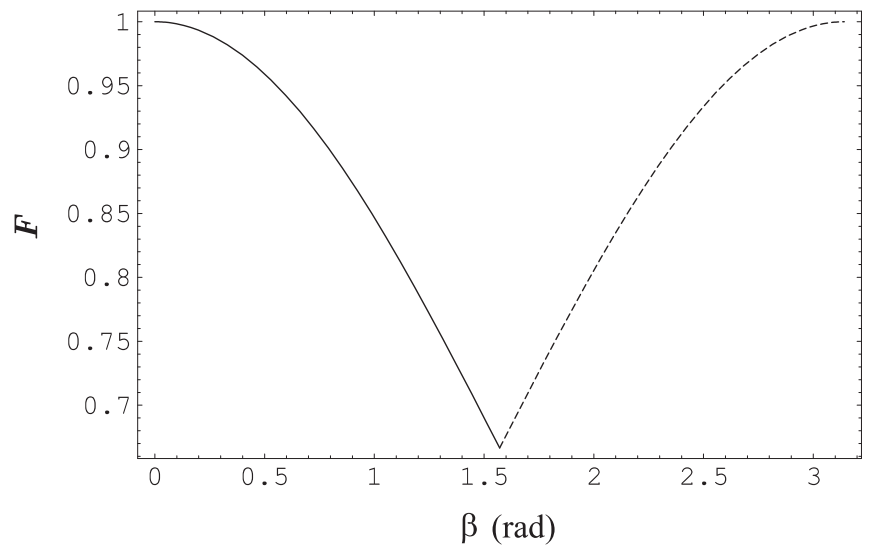

Figure 3: This is a graph for unitary transformation of nonlinear rotation when $\delta=\pi / 2$ for arbitrary $\beta$. For $0 \leq \beta \leq \pi / 2$, identity map yields the optimal map which is shown as full line. The dotted line corresponds to the rotation by $\pi$ for $\pi / 2 \leq \beta \leq \pi$.

that we have $|A\rangle=0=|\tilde{A}\rangle$ from (15). Similarly, for $\pi / 2 \leq \beta \leq \pi,|B|^{2}=1=|B|^{2}$ and $\langle B \mid \tilde{B}\rangle=-1=\langle\tilde{B} \mid B\rangle$ would give the optimal fidelity. This can be done with the choice of $|B\rangle=|0\rangle$ and $|\tilde{B}\rangle=-|0\rangle$. This latter map corresponds to the unitary rotation by $\pi$ about the $z$-axis while the former map is simply an identity map. The fidelity is given as

$$
\frac{2}{3} \leq F \leq 1
$$

where the actual dependence on the value of $\beta$ is shown in figure 3. One can easily see that when $\beta=0$ and $\beta=\pi$, it should be achieved perfectly therefore the fidelity is equal to 1 . The fidelity has the lowest value of $2 / 3$ when $\beta=\pi / 2$.

We now would like to consider a case for arbitrary $\delta$, i.e. we want

$$
\begin{cases}0 \leq \vartheta \leq \delta & \text { rotation by }+\beta \\ \delta \leq \vartheta \leq \pi & \text { rotation by }-\beta\end{cases}
$$

We consider the same output density matrix $\rho^{\text {out }}$ in (17) and the fidelity for the transformation (21) is obtained as follows,

$$
\begin{aligned}
F & =\frac{1}{4 \pi} \int_{0}^{2 \pi} \int_{0}^{\delta}\left\langle\psi(\vartheta, \varphi+\beta)\left|\rho^{\text {out }}\right| \psi(\vartheta, \varphi+\beta)\right\rangle \sin \vartheta d \vartheta d \varphi \\
& +\frac{1}{4 \pi} \int_{0}^{2 \pi} \int_{\delta}^{\pi}\left\langle\psi(\vartheta, \varphi-\beta)\left|\rho^{\text {out }}\right| \psi(\vartheta, \varphi-\beta)\right\rangle \sin \vartheta d \vartheta d \varphi
\end{aligned}
$$

Let us consider a specific example when $\beta=\pi / 3$. Then the fidelity in (22) comes out as

$$
\begin{aligned}
F= & \frac{1}{3}+\frac{1}{6}\left(|B|^{2}+|\tilde{B}|^{2}\right)+(.08333-(.1623 \cos \delta-.018 \cos 3 \delta) i)\langle B \mid \tilde{B}\rangle \\
& +(.08333+(.1623 \cos \delta-.018 \cos 3 \delta) i)\langle\tilde{B} \mid B\rangle
\end{aligned}
$$




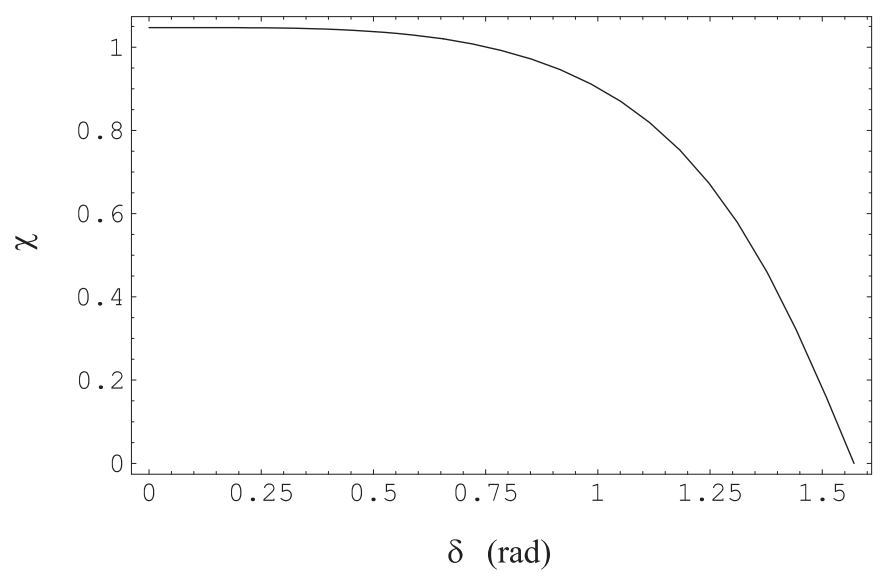

Figure 4: This is a graph between $\chi$ in (25) and $\delta$. This value of $\chi$ for a given $\delta$ gives the maximum fidelity in (22)

We need to find the values of $|B|^{2},|\tilde{B}|^{2},\langle B \mid \tilde{B}\rangle$ and $\langle\tilde{B} \mid B\rangle$ such that the fidelity $F$ yields the highest value. Suppose $\langle B \mid \tilde{B}\rangle=a+b i$ and $\langle\tilde{B} \mid B\rangle=a-b i$ where $a, b$ are real. Then the two terms of $\langle B \mid \tilde{B}\rangle$ and $\langle\tilde{B} \mid B\rangle$ in (23) are written as

$$
2(.08333) a+2(.1623 \cos \delta-.018 \cos 3 \delta) b
$$

One can verify that $(.1623 \cos \delta-.018 \cos 3 \delta)$ is non-negative for $0 \leq \delta \leq \pi / 2$, hence we know that these two terms will be maximum with an appropriate $a$ and $b$ when $a^{2}+b^{2}=1$ rather than $a^{2}+b^{2}<1$. Therefore we can choose $|B\rangle=e^{-i \chi / 2}|0\rangle$ and $|\tilde{B}\rangle=e^{i \chi / 2}|0\rangle$ which implies $\langle B \mid \tilde{B}\rangle=e^{i \chi}\left(\langle\tilde{B} \mid B\rangle=e^{-i \chi}\right)$. We can obtain $\chi$ which would give the $F$ maximum value as follows,

$$
\chi=\arccos \left(\frac{83333 . \times 10^{-3}}{\sqrt{6.94+2.63 \times 10 \cos ^{2} \delta-5.85 \cos \delta \cos 3 \delta+3.25 \times 10^{-1} \cos ^{2} 3 \delta}}\right)
$$

Therefore $\chi$ is the angle that you want to rotate by and the graph of the value $\chi$ and the angle $\delta$ is shown in figure 4 . It shows that when $\delta=0$ it is best to rotate by $\pi / 3$ and for $\delta=\pi / 2$ identity map is optimal as we have checked previously. By symmetry, the case of $\pi / 2 \leq \delta \leq \pi$ can be obtained similarly except that we rotate by opposite direction. Other cases of $\beta$ can be achieved using the similar methods described above. In figure 5 , we show the graph of fidelity and $\delta$ for different $\beta$ 's.

Therefore quantum scheme is always better than or equal to the measurement scheme (which is $F=2 / 3$ ) as expected. 


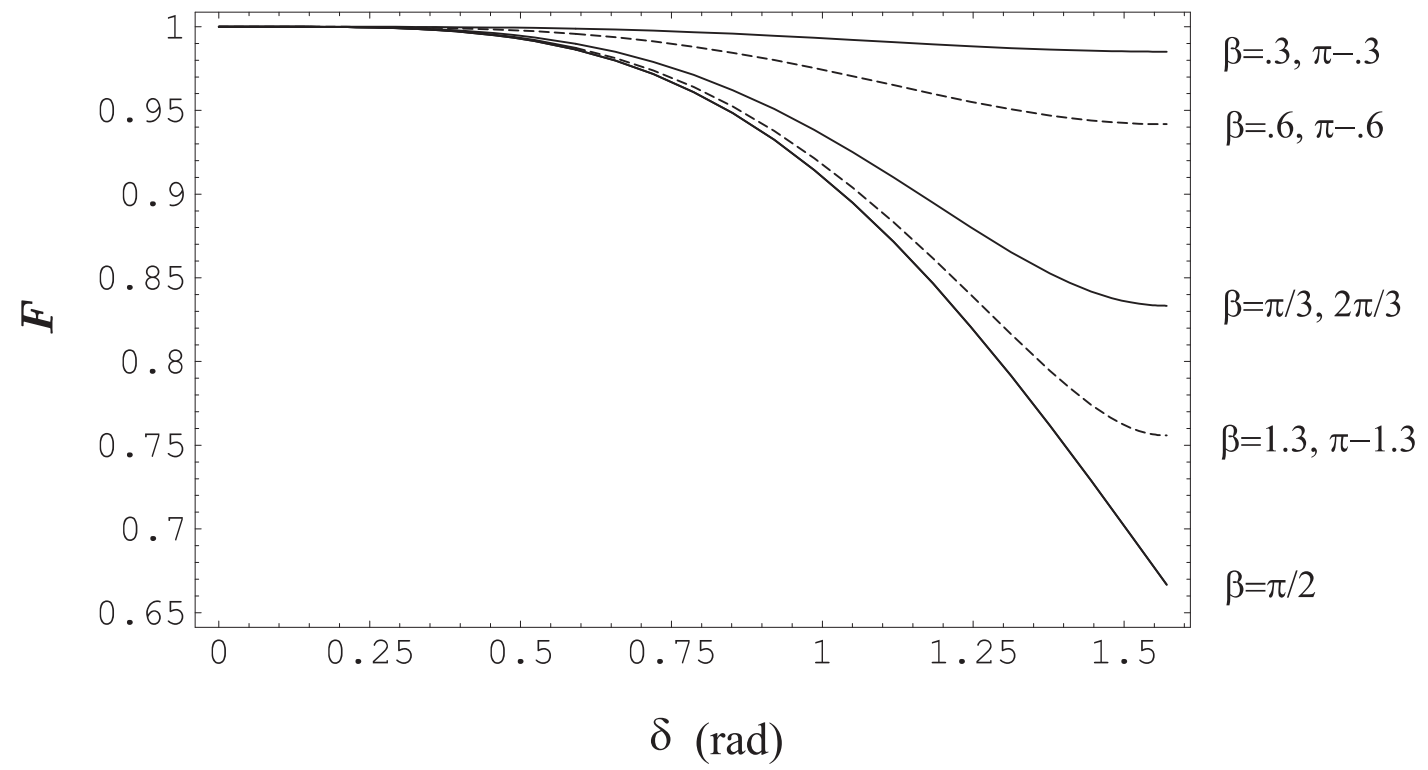

Figure 5: This graph shows the fidelities of quantum scheme for nonlinear rotation as a function of $\delta$ for different values of $\beta$. The end points of each line, i.e. at $\delta=\pi / 2$ corresponds to the graph in figure 3 .

\section{Nonlinear ORTHOG Transformations}

A NOT-gate is one of the simple operations performed on a classical bit, 0 to 1 and 1 to 0 . Just as in the cloning case, the operation which sends a qubit to its orthogonal state is not so simple. In [16, 17, 20], U-NOT operation for qubits has been studied. Since a perfect U-NOT gate would require anti-unitary operation, they considered the optimal way of imitating perfect U-NOT transformation and showed the optimal fidelity to be $2 / 3$ for a single qubit input. This fidelity was same as the measurement fidelity which we reviewed in sect. 2. Therefore measuring the qubit then preparing another qubit orthogonal to the original one and the quantum unitary transformation were shown to have equal efficiency. In this section, we generalise this result and consider nonlinear ORTHOG transformations. As shown in figure 6, we want to apply ORTHOG-gate to the shown area of a Bloch sphere, i.e.

$$
\begin{cases}0 \leq \vartheta \leq \delta, \pi-\delta \leq \vartheta \leq \pi & |\psi(\vartheta, \varphi)\rangle \rightarrow|\psi(\vartheta-\pi, \varphi)\rangle \\ \delta \leq \vartheta \leq \pi-\delta & |\psi(\vartheta, \varphi)\rangle \rightarrow|\psi(\vartheta, \varphi)\rangle\end{cases}
$$

When $\delta=0$, this transformation is simply an identity map and when $\delta=\pi / 2$, it would correspond to the U-NOT gate (if a universality condition is imposed).

Measurement Scheme In the previous sections, we considered two types of measurement schemes. We now consider these two types in achieving the transformation in 


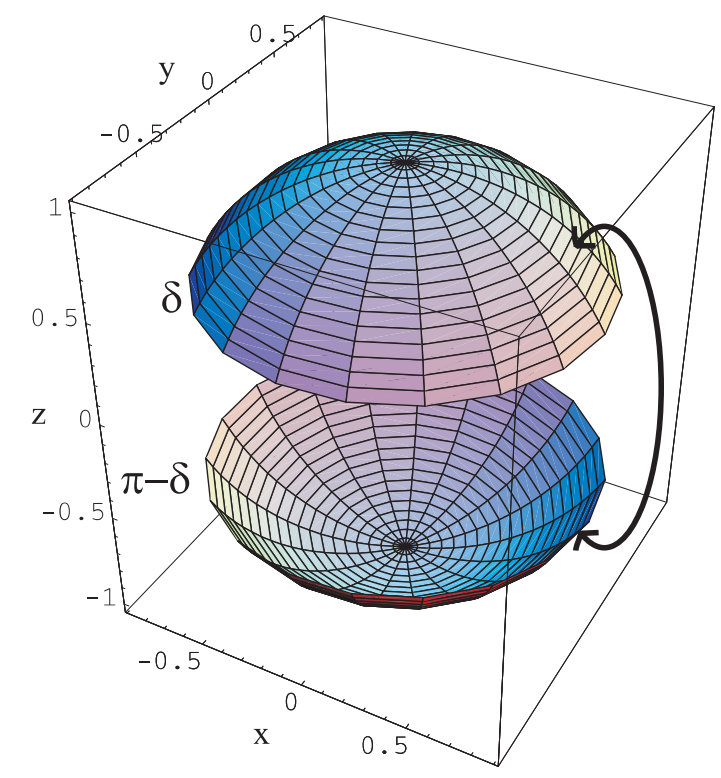

Figure 6: We want to apply ORTHOG operation only to the shown areas of a Bloch sphere, i.e. $0 \leq \vartheta \leq \delta$ and $\pi-\delta \leq \vartheta \leq \pi$ while leaving other areas of a Bloch sphere unchanged.

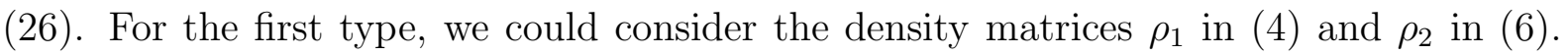
We take the average over all $|\phi\rangle$ 's then the fidelity is obtained by

$$
F_{1,2}=\int_{0}^{2 \pi} \int_{0}^{\delta}\left\langle\psi^{\perp}\left|\overline{\rho_{1,2}}\right| \psi^{\perp}\right\rangle d \Omega+\int_{0}^{2 \pi} \int_{\delta}^{\pi-\delta}\left\langle\psi\left|\overline{\rho_{1,2}}\right| \psi\right\rangle d \Omega+\int_{0}^{2 \pi} \int_{\pi-\delta}^{\pi}\left\langle\psi^{\perp}\left|\overline{\rho_{1,2}}\right| \psi^{\perp}\right\rangle d \Omega
$$

where $d \Omega=1 /(4 \pi) \sin \vartheta d \vartheta d \varphi$. Another way is to prepare $\sigma_{1}$ in (5) and $\sigma_{2}$ in (77). Although the measurement fidelity, the measurement based U-NOT and the measurement based general linear transformation all yielded the same results for both preparations of $\rho$ and $\sigma$ as shown in sect. 2, it is no longer true in case of nonlinear ORTHOG transformations. The fidelities for $\rho_{1,2}, \sigma_{1,2}$ are obtained as shown in figure 7 . Interestingly, for $0 \leq \delta \leq .82 \ldots$ $\rho_{1}$ is the better preparation while for $.82 \ldots \leq \delta \leq \pi / 2$, it is the $\sigma_{2}$ preparation which gives the higher fidelity.

Quantum Scheme We consider the same transformation (14) and the same conditions $(15,16)$. With $\rho^{\text {out }}$ in (17), the fidelity is

$$
F=\int_{0}^{2 \pi} \int_{0}^{\delta}\left\langle\psi^{\perp}\left|\rho^{\text {out }}\right| \psi^{\perp}\right\rangle d \Omega+\int_{0}^{2 \pi} \int_{\delta}^{\pi-\delta}\left\langle\psi\left|\rho^{\text {out }}\right| \psi\right\rangle d \Omega+\int_{0}^{2 \pi} \int_{\pi-\delta}^{\pi}\left\langle\psi^{\perp}\left|\rho^{\text {out }}\right| \psi^{\perp}\right\rangle d \Omega
$$

where $d \Omega=1 /(4 \pi) \sin \vartheta d \vartheta d \varphi$. Let us consider the simplest case when $\delta=\pi / 2$ which corresponds to the ORTHOG gate. In that case, the fidelity in (28) becomes

$$
F=\frac{1}{3}|A|^{2}+\frac{1}{3}|\tilde{A}|^{2}+\frac{1}{6}\left(|B|^{2}+|\tilde{B}|^{2}-\langle B \mid \tilde{B}\rangle-\langle\tilde{B} \mid B\rangle\right)
$$




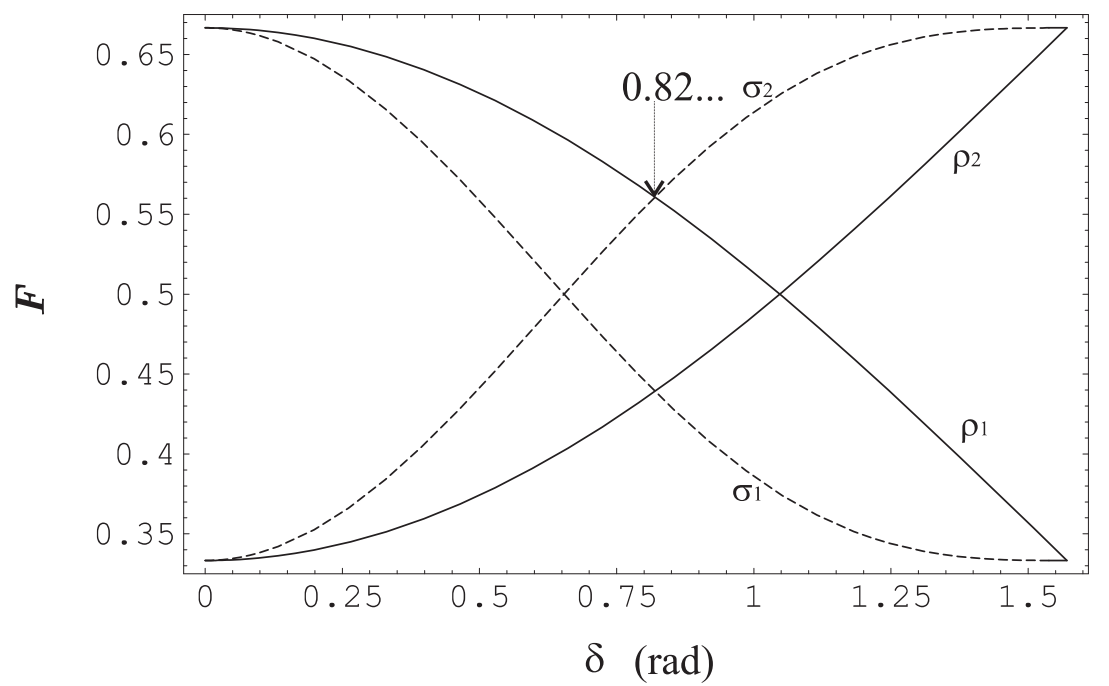

Figure 7: $\rho_{1}$ and $\rho_{2}$ are represented by full lines and the dotted lines represent $\sigma_{1}$ and $\sigma_{2}$. For $0 \leq \delta \leq .82 \ldots, \rho_{1}$ is the optimal preparation while for $.82 \ldots \leq \delta \leq \pi / 2$, it is the $\sigma_{2}$ which gives the higher fidelity.

$$
=\frac{2}{3}-\frac{1}{6}\left(|B|^{2}+|\tilde{B}|^{2}+\langle B \mid \tilde{B}\rangle+\langle\tilde{B} \mid B\rangle\right)
$$

Suppose $|B\rangle=(a+b i)|0\rangle$ and $|\tilde{B}\rangle=(c+d i)|0\rangle$ where $a, b, c, d$ are real. This implies

$$
|B|^{2}=a^{2}+b^{2},|\tilde{B}|^{2}=c^{2}+d^{2}
$$

then

$$
|B|^{2}+|\tilde{B}|^{2}+\langle B \mid \tilde{B}\rangle+\langle\tilde{B} \mid B\rangle=(a+c)^{2}+(b+d)^{2}
$$

This quantity is always non-negative and the minimum is achieved with $a=-c$ and $b=-d$ therefore

$$
|B\rangle=-|\tilde{B}\rangle,|A|^{2}=|\tilde{A}|^{2}
$$

What if $|B\rangle$ and $|\tilde{B}\rangle$ are not the vectors pointing in the same direction? Since $|B|^{2}+|\tilde{B}|^{2}+$ $\langle B \mid \tilde{B}\rangle+\langle\tilde{B} \mid B\rangle=|| B\rangle+\left.|\tilde{B}\rangle\right|^{2} \geq 0$, we know (33) is optimal. The U-NOT transformation derived in [16, 17] is

$$
|A\rangle=-\sqrt{\frac{2}{3}}|00\rangle,|\tilde{A}\rangle=\sqrt{\frac{2}{3}}|11\rangle,|B\rangle=\sqrt{\frac{1}{6}}(|01\rangle+|10\rangle),|\tilde{B}\rangle=-\sqrt{\frac{1}{6}}(|01\rangle+|10\rangle)
$$

This is one special case of (33) which is consistent considering the universality of (34).

For arbitrary $\delta$, the fidelity is obtained using the formula in (28) which is given as

$$
F=X+Y\left(|B|^{2}+|\tilde{B}|^{2}\right)+Z(\langle B \mid \tilde{B}\rangle+\langle\tilde{B} \mid B\rangle)
$$




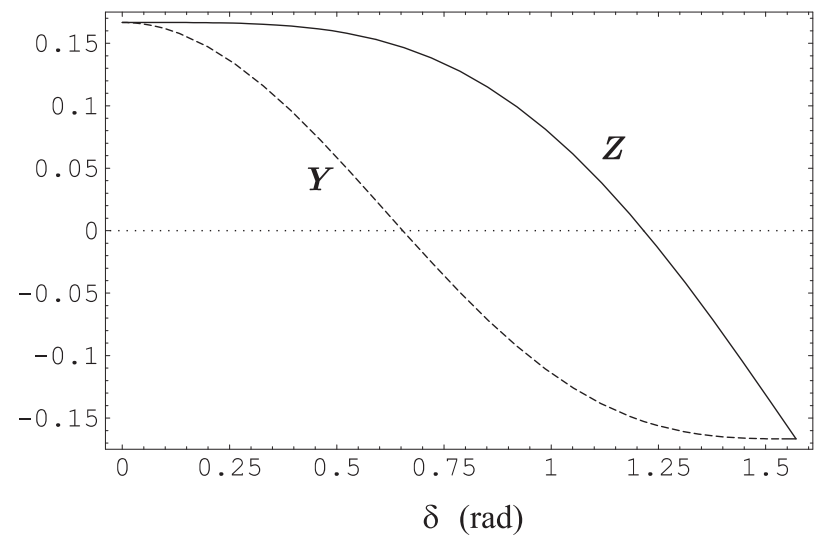

Figure 8: This shows the coefficients $Y$ and $Z$ in (37) and (38) as a function of $\delta$.

where

$$
\begin{aligned}
X & \equiv \frac{2}{3}+\frac{1}{24}(\cos 3(\pi-\delta)-\cos 3 \delta)-\frac{1}{4} \cos \delta \\
Y & \equiv-\frac{1}{6}-\frac{1}{8}(\cos 2(\pi-\delta)-\cos 2 \delta)-\frac{1}{24}(\cos 3(\pi-\delta)-\cos 3 \delta)+\frac{1}{4} \cos \delta \\
Z & \equiv-\frac{1}{6}+\frac{1}{48}(\cos 3(\pi-\delta)-\cos 3 \delta)+\frac{3}{8} \cos \delta
\end{aligned}
$$

We would like to find the values of $|B|^{2},|\tilde{B}|^{2},\langle B \mid \tilde{B}\rangle$ and $\langle\tilde{B} \mid B\rangle$ such that the fidelity in (35) is maximum. For $0 \leq \delta \leq \pi / 2, X$ is always positive. The values of $Y$ and $Z$ as functions of $\delta$ are shown in figure 8 . When $Y \geq 0$ and $Z \geq 0$, then the optimal map can be obtained when $|B\rangle=|0\rangle=|\tilde{B}\rangle$ which is simply an identity map.

Let us consider the case when $Y<0$ and $Z>0$ in particular when $|Y| \leq|Z|$. As before, suppose $|B\rangle=(a+b i)|0\rangle$ and $|\tilde{B}\rangle=(c+d i)|0\rangle$ where $a, b, c, d$ are real numbers. Then

$$
\begin{aligned}
& Y\left(B^{2}+\tilde{B}^{2}\right)+Z(\langle B \mid \tilde{B}\rangle+\langle\tilde{B} \mid B\rangle) \\
= & Y\left(a^{2}+b^{2}+c^{2}+d^{2}\right)+Z(2 a c+2 b d) \\
= & \left.Y\left((a-c)^{2}+(b-d)^{2}\right)\right)+(Z-Y)(2 a c+2 b d)
\end{aligned}
$$

Since $Y \leq 0, a=c$ and $b=d$ will give the $Y$ term in (40) highest, which is zero. Let us consider the term $(a c+b d)$. Since we are assuming $|B\rangle$ and $|\tilde{B}\rangle$ may not be normalised, we know $a^{2}+b^{2} \leq 1$ and $c^{2}+d^{2} \leq 1$. We know that the highest value for $a c+b d$ will be attained when $a^{2}+b^{2}=1$ and $c^{2}+d^{2}=1$. Let us consider

$$
\begin{aligned}
& a c+b d \stackrel{?}{\leq} 1 \\
\Rightarrow \quad & a c+\sqrt{\left(1-a^{2}\right)\left(1-c^{2}\right)} \leq 1
\end{aligned}
$$




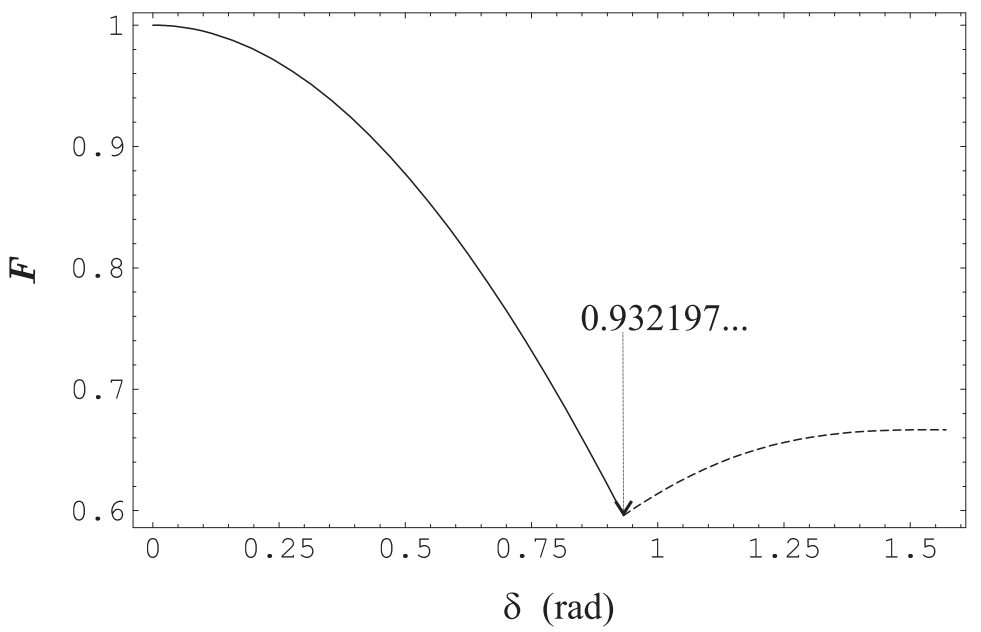

Figure 9: This is a graph for nonlinear ORTHOG transformation. It shows the fidelity $F$ as a function of $\delta$ where the full line is an identity map and the dotted line represents the unitary operation of Pauli matrix $\sigma_{x}$.

$$
\begin{aligned}
& \Rightarrow \quad\left(1-a^{2}\right)\left(1-c^{2}\right) \leq(1-a c)^{2} \\
& \Rightarrow \quad 0 \leq a^{2}-2 a c+c^{2}=(a-c)^{2}
\end{aligned}
$$

Therefore from (44), we know (41) is true. Therefore the highest possible value for the $(Z-Y)$ term is achieved when $a=c$ (which we choose to be 1 ) and this also gives the lowest possible value for the $Y$ term. If $B$ and $\tilde{B}$ were the vectors pointing different directions, the second term in (39) which is positive will be even less. Therefore our choice of the same state $|0\rangle$ (or any other normalised state) for $|B\rangle$ and $|\tilde{B}\rangle$ is indeed optimal.

Let us consider the case when $|Y| \geq|Z|$ for $Y<0$ and $Z>0$. Since $a^{2}+c^{2} \geq \pm 2 a c$ and $b^{2}+d^{2} \geq \pm 2 b d$, we know (39) is non-positive. Therefore the choice of $|B\rangle=0=|\tilde{B}\rangle$ yields the highest fidelity. For a region where $Y<0$ and $Z<0$, one can see $|Y|>|Z|$ from figure 8. Therefore again $|B\rangle=0=|\tilde{B}\rangle$ is the optimal map. This map corresponds to the transformation $|0\rangle \rightarrow|1\rangle,|1\rangle \rightarrow|0\rangle$ which is one of the Pauli matrices $\sigma_{x} \equiv\left(\begin{array}{ll}0 & 1 \\ 1 & 0\end{array}\right)$. The final optimal map is obtained as follows,

$$
\begin{cases}0 \leq \delta \leq .932197 \ldots & \mathbf{1} \\ .932197 \ldots \leq \delta \leq \pi / 2 & |0\rangle \rightarrow|1\rangle,|1\rangle \rightarrow|0\rangle\end{cases}
$$

where $\delta=.932197 \ldots$ corresponds to the point when $|Y|=|Z|$ for the period $Y<0$ and $Z>0$. Therefore the optimal map is either an identity map or a $\sigma_{x}$ unitary operation depending on the value of $\delta$. 

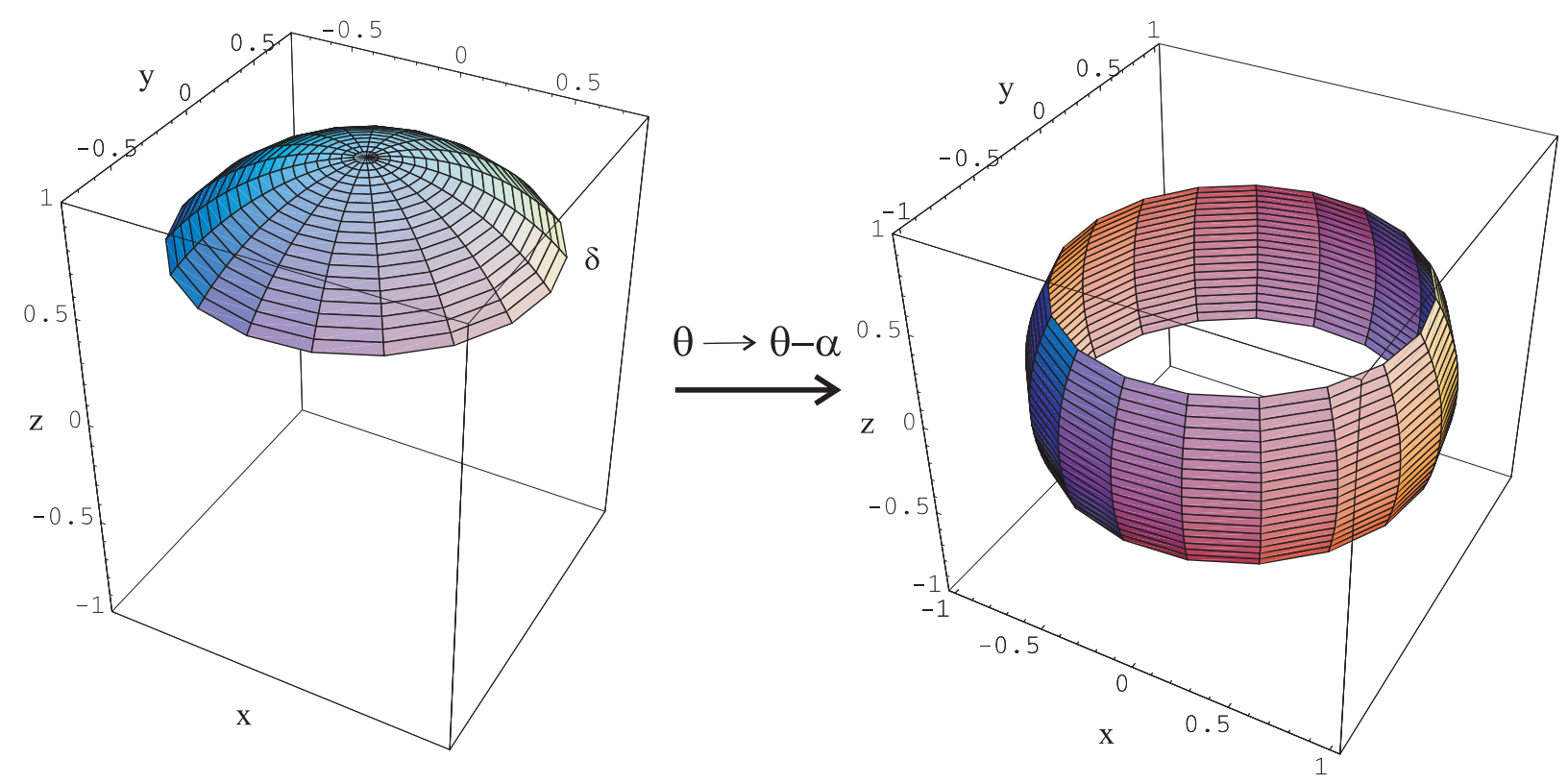

Figure 10: This figure shows the nonlinear general transformation. We want to transform the shown area of a Bloch sphere by $(\vartheta, \varphi) \rightarrow(\vartheta-\alpha, \varphi)$ while leaving other areas of a Bloch sphere unchanged.

\section{Nonlinear General Transformations}

Motivated by the U-NOT gate, we investigated a general arbitrary manipulation that can be performed on a single qubit by studying the universal linear transformation $(\vartheta, \varphi) \rightarrow$ $(\vartheta-\alpha, \varphi)$ in [19]. Rather surprisingly, we have found there are only two maps which optimise such transformation, either identity or U-NOT gate depending on $\alpha$. In this section, we attempt to generalise this result to nonlinear cases. As shown in figure 10, we want to transform $|\psi(\vartheta, \varphi)\rangle$ into $|\psi(\vartheta-\alpha, \varphi)\rangle$ only when $\vartheta$ is between 0 and an arbitrary angle $\delta$, i.e.,

$$
\left\{\begin{array}{l}
0 \leq \vartheta \leq \delta, \quad|\psi(\vartheta, \varphi)\rangle \rightarrow|\psi(\vartheta-\alpha, \varphi)\rangle \\
\delta<\vartheta \leq \pi, \quad|\psi(\vartheta, \varphi)\rangle \rightarrow|\psi(\vartheta, \varphi)\rangle
\end{array}\right.
$$

When $\delta=\pi$, it would correspond to what we have already considered for the universal transformation in [19].

Measurement Scheme As in the nonlinear ORTHOG transformation case, we consider the same density matrices $\rho_{1,2}$ in (4,6) and $\sigma_{1,2}$ in (5, (7). We also take two examples of $\alpha, \alpha=\pi / 3$ and $\alpha=2 \pi / 3$. Then the fidelity is obtained as follows,

$$
F_{1,2}=\int_{0}^{\delta}\left\langle\psi(\vartheta-\alpha, \varphi)\left|\overline{\rho_{1,2}}\right| \psi(\vartheta-\alpha, \varphi)\right\rangle d \Omega+\int_{\delta}^{\pi}\left\langle\psi(\vartheta, \varphi)\left|\overline{\rho_{1,2}}\right| \psi(\vartheta, \varphi)\right\rangle d \Omega
$$



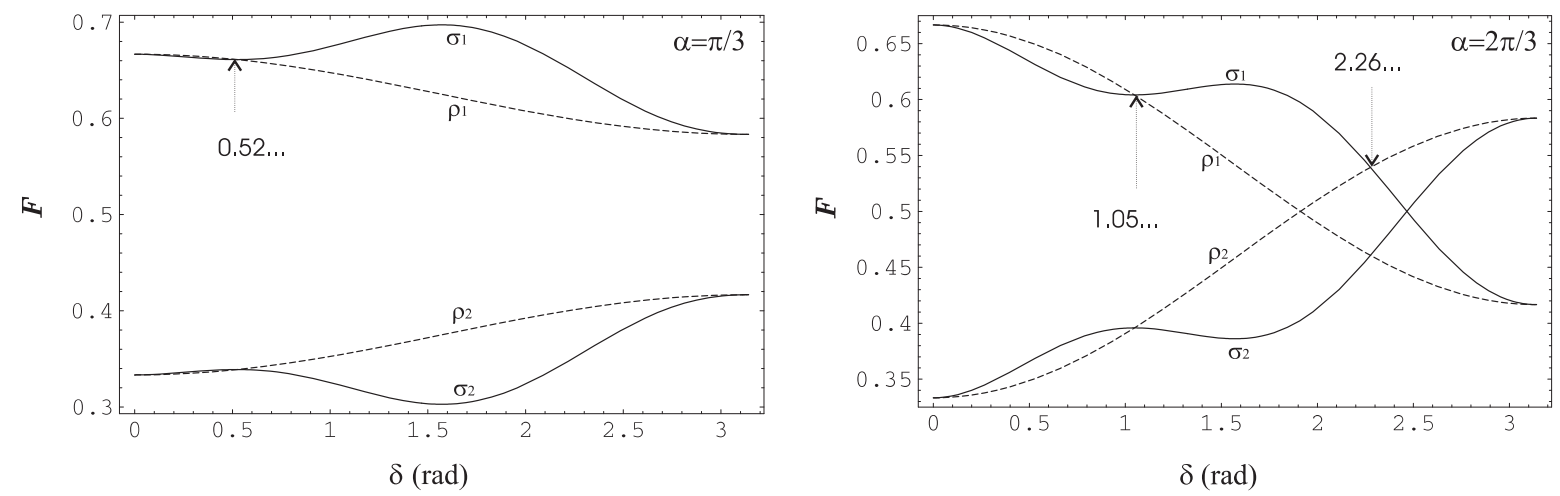

Figure 11: These two graphs show the fidelity of measurement scheme for nonlinear general transformations. The left graph is for $\alpha=\pi / 3$ and the right one is when $\alpha=2 \pi / 3$. The full lines are for $\sigma_{1,2}$ and the dotted lines represent $\rho_{1,2}$.

where $0 \leq \delta \leq \pi$ and similarly for $\sigma_{1,2}$. As in the nonlinear ORTHOG case, these two preparations yield different results that are shown in figure 11. Therefore the following maps have higher fidelities at least among the measurement preparations, $\rho_{1,2}$ and $\sigma_{1,2}$,

$$
\begin{cases}0 \leq \delta \leq 0.52 \ldots, & \rho_{1} \\ 0.52 \ldots \leq \delta \leq \pi, & \sigma_{1}\end{cases}
$$

for $\alpha=\pi / 3$ and

$$
\begin{cases}0 \leq \delta \leq 1.05 \ldots, & \rho_{1} \\ 1.05 \ldots \leq \delta \leq 2.26 \ldots, & \sigma_{1} \\ 2.26 \ldots \leq \delta \leq \pi, & \rho_{2}\end{cases}
$$

when $\alpha=2 \pi / 3$. Let us now investigate a quantum case.

Quantum Scheme Let us first consider the case which we showed in our previous paper [19], i.e. the transformation in (46) and we take $\delta$ to be $\pi$. Then the fidelity is obtained as follows,

$$
\begin{aligned}
F & =\int_{0}^{\delta=\pi}\left\langle\psi(\vartheta-\alpha, \varphi)\left|\rho^{\text {out }}\right| \psi(\vartheta-\alpha, \varphi)\right\rangle d \Omega \\
& =V+X|B|^{2}+Y|\tilde{B}|^{2}+Z(\langle B \mid \tilde{B}\rangle+\langle\tilde{B} \mid B\rangle)
\end{aligned}
$$

where

$$
\begin{aligned}
V & =\frac{1}{2}-\frac{1}{6} \cos \alpha, \quad X=\frac{1}{6} \cos \alpha+.3926 \sin \alpha \\
Y & =\frac{1}{6} \cos \alpha-.3926 \sin \alpha, \quad Z=\frac{1}{6} \cos \alpha
\end{aligned}
$$

The values of $V, X, Y$ and $Z$ as functions of $\delta$ are shown in figure 12. Since $V$ is always positive for all $\alpha$, let us consider the other three terms. When $X, Y$ are both positive, 


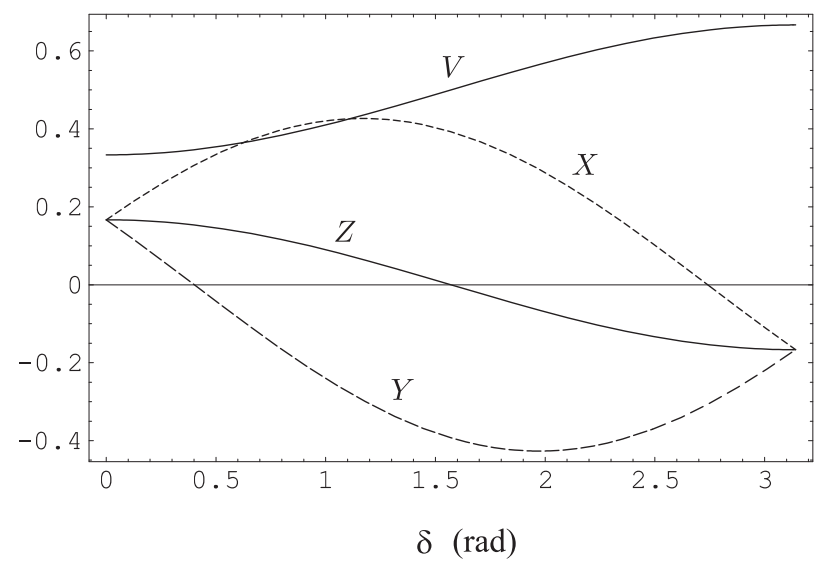

Figure 12: This figure shows the coefficients $V, X, Y, Z$ in (52,53) as a function of $\delta$.

then the maximum $F$ is obtained when the $|B|^{2}=|\tilde{B}|^{2}=\langle B \mid \tilde{B}\rangle=\langle\tilde{B} \mid B\rangle=1$ which can be achieved easily with the choice of $|B\rangle=|\tilde{B}\rangle=|0\rangle$, for instance.

We consider the case when $X, Y$ are both negative. As before we assume $|B\rangle=$ $(a+b i)|0\rangle$ and $|\tilde{B}\rangle=(c+d i)|0\rangle$. Then (51) is rewritten as follows

$$
V+X\left(a^{2}+b^{2}\right)+Y\left(c^{2}+d^{2}\right)+Z(2 a c+2 b d)
$$

Since the $Z$ term can always be non-negative with an appropriate sign choices of $a, b, c, d$, we do not need to worry about case of different vectors (i.e. different directions) for $|B\rangle$ and $|\tilde{B}\rangle$ which would only lessen the value of $Z$ term. The $X, Y$ and $Z$ terms in (54) can be rewritten as follows,

$$
\begin{aligned}
& X a^{2}+Y c^{2}+Z(2 a c)+X b^{2}+Y d^{2}+Z(2 b d) \\
= & Y\left(c+\frac{Z}{Y} a\right)^{2}+Y\left(d+\frac{Z}{Y} b\right)^{2}+\left(X-\frac{Z^{2}}{Y}\right)\left(a^{2}+b^{2}\right)
\end{aligned}
$$

One can check that $\left(X-Z^{2} / Y\right)>0$ for the region where $X, Y<0$. Therefore with the choice of $a=1, b=0$ and $c=-Z / Y, d=0$, the fidelity will be the highest.

Let us consider the case when $X>0$ and $Y<0$. In this case, we could consider (56) again. The first two terms are always non-positive and the second term is non-negative because $X-Z^{2} / Y>0$ for the region where $X>0, Y<0$. Then $a=1$ and $c=-Z / Y$ would yield the optimal fidelity. However $|Z / Y|>1$ when $Y$ is near 0 . We then consider the equation in (54) again. Since $X$ is positive, we know $a^{2}+b^{2}=1$ would yield the highest fidelity. Therefore we have (without the $V$ term),

$$
X+Y\left(c^{2}+d^{2}\right)+Z\left(2 a c+2 \sqrt{1-a^{2}} d\right)
$$

and we assume $a, c, d$ to be non-negative. By taking partial derivatives, we can obtain the maximum value. In this case, it is the end point, $c=1$ (and $a=1$ ) that gives the optimal 


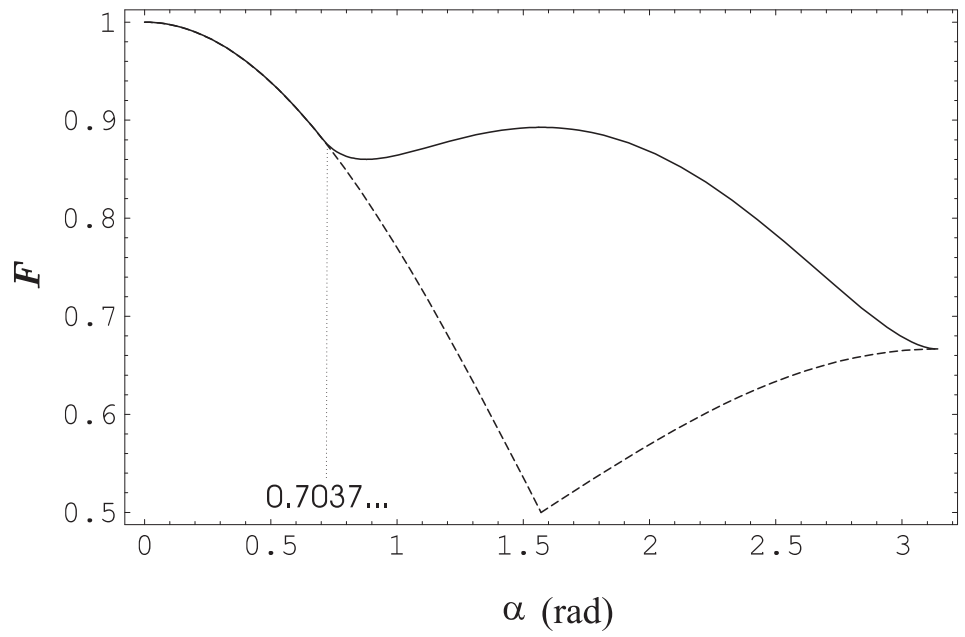

Figure 13: This figure shows the fidelities as a function of $\alpha$ when $\delta=\pi$. The dotted line is for universal general linear transformation as shown in [19] and the full line represents the averaged general linear transformation which has substantially higher fidelity for $\alpha \geq$ $.7037 \ldots$.

fidelity. The final graph can be obtained as shown in figure 13. Note that unlike the case of nonlinear ORTHOG transformation (where the optimal map was either identity or $\sigma_{x}$ ), the mapping is continuous for some regions. It shows that the fidelity of averaged general transformation (full line) is higher than the universal general operation case (dotted line) that was obtained in [19] for $\alpha \geq .7037 \ldots$.

Next we consider the proposed map in (46) for arbitrary $\delta$. The fidelity is then

$$
F=\int_{0}^{\delta}\left\langle\psi(\vartheta-\alpha, \varphi)\left|\rho^{\text {out }}\right| \psi(\vartheta-\alpha, \varphi)\right\rangle d \Omega+\int_{\delta}^{\pi}\left\langle\psi(\vartheta, \varphi)\left|\rho^{\text {out }}\right| \psi(\vartheta, \varphi)\right\rangle d \Omega
$$

Using a similar method as in the case of $\delta=\pi$ we considered above, we can obtain the optimal fidelities as shown in figure 14. In the figure, we show five different values of $\alpha$ where the end point of each line (i.e. when $\delta=\pi$ ) corresponds to the full line we obtained in figure 13 .

\section{Remarks}

In this paper, we have studied three types of nonlinear operations. Firstly, we considered nonlinear rotations that rotate different parts of a Bloch sphere (i.e. $0 \leq \vartheta \leq \delta$ and $\delta \leq \vartheta \leq \pi)$ in opposite directions about the $z$-axis. In case of a measurement-based scheme, we showed that a measurement onto the particular (rather than arbitrary) basis, $\{|0\rangle,|1\rangle\}$, gives $2 / 3$ fidelity. For a quantum unitary transformation, we considered average rotation manipulations. We showed that when the rotations are applied to upper and lower 


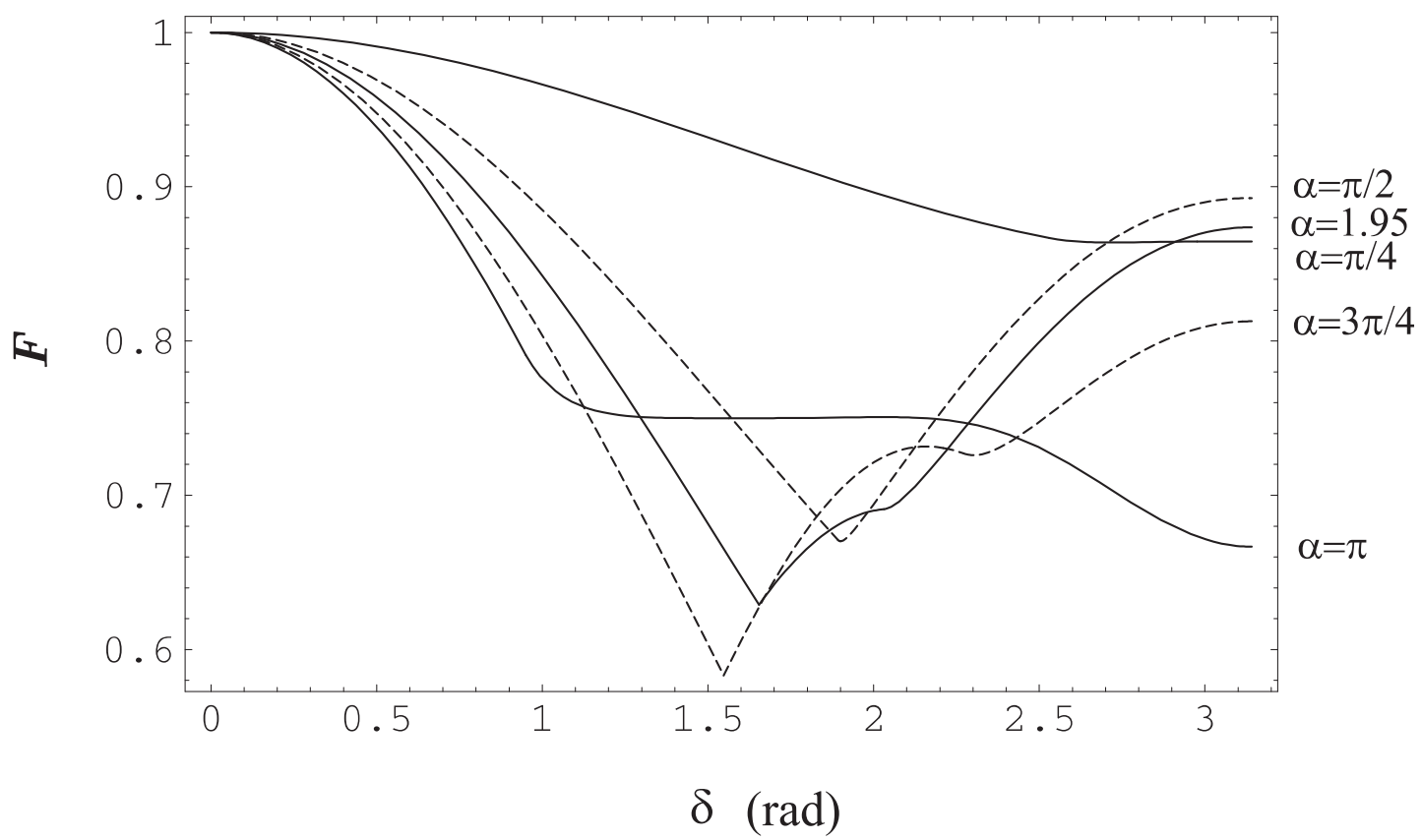

Figure 14: This is graph of nonlinear general transformations. The fidelities for different values of $\alpha$ as functions of $\delta$ are shown. The end points of each line (i.e. when $\delta=\pi$ corresponds to the full line in figure 13 .

hemispheres of a Bloch sphere (i.e. when $\delta=\pi / 2$ ), the optimal map is either identity (for $0 \leq \beta \leq \pi / 2$ ) or the unitary rotation by $\pi$ (for $\pi / 2 \leq \beta \leq \pi$ ). For general $\delta$ 's, we found the optimal maps for different values of the rotating angle $\beta$. In these cases, the optimal operations correspond to unitary rotations by $\chi(\delta)$ about the $z$-axis.

Secondly, we studied nonlinear ORTHOG transformations where the operations were applied only to $0 \leq \vartheta \leq \delta$ and $\pi-\delta \leq \vartheta \leq \pi$. For a measurement scheme, we found the preparations $\rho$ 's and $\sigma$ 's yield different results. For $0 \leq \delta \leq .82 \ldots, \rho_{1}$ in (4) yields a higher fidelity while for $.82 \ldots \leq \delta \leq \pi / 2, \sigma_{2}$ in (7) is a better preparation. In case of a quantum scheme, we considered a special case when the ORTHOG operation is applied to a whole Bloch sphere (i.e. when $\delta=\pi / 2$ ). In this case, we found Bužek et al.'s U-NOT gate appears as a special case of average ORTHOG transformation when universality is imposed. For arbitrary $\delta$, we showed the optimal maps are either identity (when $0 \leq \delta \leq .932 \ldots$ ) or the Pauli matrix $\sigma_{x}$ (when $.932 \ldots \leq \delta \leq \pi / 2$ ).

Lastly, we considered general transformations, i.e. $(\vartheta, \varphi) \rightarrow(\vartheta-\alpha, \varphi)$, that are applied only to a partial area of a Bloch sphere $(0 \leq \vartheta \leq \delta)$. In a measurement scheme, we considered four different preparations, $\rho_{1,2}$ and $\sigma_{1,2}$ when $\alpha=\pi / 3$ and $2 \pi / 3$. For a quantum scheme, we found that, in general, the average general transformation yields higher fidelities than the universal general linear maps studied in [19]. We also found 
optimal nonlinear general maps for different values of $\alpha$.

\section{Acknowledgments}

We are grateful to Mark Hillery for helpful discussions. L.H. acknowledges support from the Royal Society.

\section{References}

[1] A. Ekert and R. Jozsa, Rev. Mod. Phys. 68, 733 (1996).

[2] A.M. Steane, Rep. Prog. Phys. 61, 117 (1998).

[3] V. Vedral and M.B. Plenio, Prog. Quant. Eletron. 22, 1 (1998).

[4] V. Bužek and M. Hillery, Phys. Rev. A 54, 1844 (1996).

[5] V. Bužek and M. Hillery, Phys. Rev. Lett. 81, 5003 (1998).

[6] N. Gisin, and S. Massar, Phys. Rev. Lett. 79, 2153 (1997).

[7] R.F. Werner, Phys. Rev. A 58, 1827 (1998).

[8] D. Bruß, A. Ekert, and C. Michiavello, Phys. Rev. Lett. 81, 2598 (1998).

[9] D. Bruß, D. DiVincenzo, A. Ekert, C.A. Fuchs, C. Michiavello, and J.A. Smolin, Phys. Rev. A 57, 2368 (1998).

[10] V. Bužek and M. Hillery, Phys. Rev. A 62, 022303 (2000).

[11] D.R. Terno, Phys. Rev. A 59, 3320 (1999).

[12] T. Mor and D.R. Terno, Phys. Rev. A 60, 4341 (1999).

[13] T. Mor, Phys. Rev. Lett. 83, 1451 (1999).

[14] S. Ghosh, S. Bandyopadhyay, A. Roy, D. Sarkar, and G. Kar, Phys. Rev. A 61, $052301(2000)$.

[15] V. Bužek and M. Hillery, Phys. Rev. A 62, 052303 (2000).

[16] V. Bužek, M. Hillery, and F. Werner, Phys. Rev. A 60, R2626 (1999).

[17] V. Bužek, M. Hillery, and F. Werner, J. Mod. Opt. 47, 2112 (2000).

[18] N. Gisin and S. Popescu, Phys. Rev. Lett. 83, 432 (1999). 
[19] L. Hardy and D.D. Song, Phys. Rev. A 63, 032304 (2001).

[20] N. Gisin, (in a private conversation).

[21] S. Massar and S. Popescu, Phys. Rev. Lett. 74, 1259 (1995).

[22] R. Derka, V. Bužek, and A. Ekert, Phys. Rev. Lett. 80, 1571 (1998). 